\title{
Numerical Determination of Orthotropic Material Properties of Textile Composite Layers and their Validation by Measurement
}

\author{
Gergely Bojtár ${ }^{1}$, Béla M. Csizmadia ${ }^{2}$, János Égert ${ }^{1}$ \\ ${ }^{1}$ Széchenyi István University, Department of Applied Mechanics, Egyetem tér 1, \\ H-9026 Győr, Hungary, bojtar@sze.hu, egert@sze.hu \\ ${ }^{2}$ Szent István University, Institute of Mechanics and Machinery, Páter K. u. 1, \\ H-2103 Gödöllö, Hungary, csizmadia.bela@gek.szie.hu
}

\begin{abstract}
The knowledge of orthotropic material properties of composite layers is very important for mechanical design of machine structures from composite material. This information cannot be found in related professional literature therefore it is necessary to determine by measurement, by basic experiments of mechanics of materials. The paper presents a new $3 D$ finite element layer model-cell based on the meso-structure of a textile composite layer, with which one shall be able to determine the material constants of an orthotropic layer. The applicability of the numerical layer model-cell and the accuracy of the numerical results are confirmed by experimental results. The numerically determined material properties of the layers are used at mechanical modeling and computation of complex, layered composite structures.
\end{abstract}

Keywords: orthotropic material properties; textile composite; composite layer; finite element method, layer model-cell; validation with experiments

\section{Introduction}

Textile composites are plastics reinforced by glass, carbon, aramid, etc. fabrics. The laminated textile composite plates consist of one 2D fabric in each layer. The reinforcing fibers are arranged into flat roving, that run parallel to each other in the textile. The thickness of the textile and a composite layer can be measured in tenth of mm-s. The mechanical analysis of a textile composite structure is a complicated problem because every single layer and the roving in it have anisotropic feature generally. With the majority of engineering problems the behavior of the material can be described by homogeneous macroscopic modeling with satisfying accuracy from engineering point of view. There is a verified computation method for determination of the material properties of a multilayered complete plate from 
given layer material properties. The aim of this research is to provide a new, improved numerical method, a layer model-cell for computation of material properties of a single layer.

Different kinds of fabrics are used in textile composites and the majority of them have two main roving directions, perpendicular to each other. These main or principal fiber directions make it possible that the behavior of the majority of textile composite materials can be handled as an orthotropic material from macroscopic point of view with sufficient accuracy.

In Figure 1 it is seen the $x_{1} x_{2}$ material coordinate system of the textile composite layer. $x_{1}$ is the chain direction (first principal material direction) of the textile whereas $x_{2}$ is the weft direction (second principal material direction).

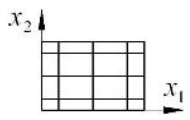

Figure 1

Material coordinate system of a textile composite layer

From macroscopic point of view a textile composite layer can be considered as an orthotropic material in the plane stress state with good approximation. The linear elastic, orthotropic material law in case of the plane stress state, in the coordinate system of principal material directions of the layer, has the following form [4], [9]:

$$
\begin{gathered}
\overline{\bar{\sigma}}=\underline{\underline{C}} \underline{\underline{\varepsilon}}, \\
{\left[\begin{array}{c}
\bar{\sigma}_{1} \\
\bar{\sigma}_{2} \\
\bar{\tau}_{12}
\end{array}\right]=\frac{1}{1-v_{12} v_{21}}\left[\begin{array}{ccc}
E_{1} & v_{21} E_{1} & 0 \\
v_{12} E_{2} & E_{2} & 0 \\
0 & 0 & \left(1-v_{12} v_{21}\right) G_{12}
\end{array}\right]\left[\begin{array}{l}
\bar{\varepsilon}_{1} \\
\bar{\varepsilon}_{2} \\
\bar{\gamma}_{12}
\end{array}\right] .}
\end{gathered}
$$

Material law (1) of the textile composite layer contains five macroscopic material constants: the $E_{1}, E_{2}$ moduli of elasticity in principal material directions, the $v_{12}$, $v_{21}$ Poisson's ratios and the $G_{12}$ shear modulus of elasticity. Four of them are independent because the matrix $\underset{\underline{C}}{C}$ of material constants is symmetric, therefore the relationship between the Young's moduli and Poisson's ratios is [4]:

$$
\frac{v_{12}}{E_{1}}=\frac{v_{21}}{E_{2}}
$$

The average strain and stress of a textile composite layer with Volume $V$ can be defined as follows [11], [12], [14]:

$\bar{\varepsilon}_{i j}=\frac{1}{V} \int_{(V)} \varepsilon_{i j} d V, \quad \bar{\sigma}_{i j}=\frac{1}{V} \int_{(V)} \sigma_{i j} d V$. 
There are no standards for building of the structure of a textile composite, for the fiber/matrix proportion and coupling and for the weaving method therefore there are a number of ways for the composite material variations and engineering applications. Therefore, the material properties of a composite structure usually cannot be found in the literature, in manuals or in standards. For determination of the material constants of a textile composite there are usually two procedures:

- In the first one, the given material is produced and the material properties are determined by measurement on test specimens cut out from the material.

- In the other one, the material constants of a single composite layer are determined by a so-called mixture rule knowing the material constants and volume fraction of the fiber and the matrix in the composite layer.

The aim of our research is to provide a numerical method for determination of the material constants in the material law (1). For this purpose, we developed a finite element 3D layer model-cell. This model-cell is suitable for the determination of the macroscopic material constants of a textile composite layer of known geometry and material parts. We have proved by measurements on eight-layer composite test specimens that the macroscopic material constants computed by the layer model-cell can reach the necessary accuracy for the modeling of the structure. The paper describes the necessary steps for building of the layer model-cell and the prescription of the loading and the periodic boundary conditions for the given loading cases in general. It shows how to calculate the material constants from the numerical results.

For determination of material properties of the layer there are several numerical methods in the relevant literature which use the material properties of the textile and matrix of the layer [10], [15], [16], [17]. Compared to the models that can be found in the literature we specified the kinematic boundary conditions in a different way in this paper. In addition, we defined the average stress of the finite element model-cell also in a different way. From these modifications, we hope the improvement and growth of the accuracy of the layer model-cell.

\section{The Roving Model-Cell}

A roving in the textile includes several thousands of $\mu \mathrm{m}$ diameter reinforcing fibers which are impregnated by the matrix material. There is also matrix material between the single fibers. We do not model these single fibers separately in the layer model-cell of the textile composite. We consider the roving as one "fiber" if we model the layer reinforced by textile woven from homogeneous roving's.

The cross section and the $x_{r 1} x_{r 2} x_{r 3}$ roving material coordinate system of the investigated roving, which is impregnated by matrix, can be seen in Figure 2 . In 
figures the length dimensions are given in $\mathrm{mm}$. The direction parallel to the reinforcing fibers is $x_{r 1}$. We determined the orthotropic, macroscopic material properties of the roving by the roving model-cell described in the paper [3]. For the structure of the roving model cell we assume that the reinforcing fibers are positioned in a regular hexagonal shape everywhere in the complete roving. In a hexagonal layout (Figure 3) one fiber is surrounded by six others in a way that those are in equal distance from each other. The cross section of the roving model-cell is marked by a thick line in Figure 3.

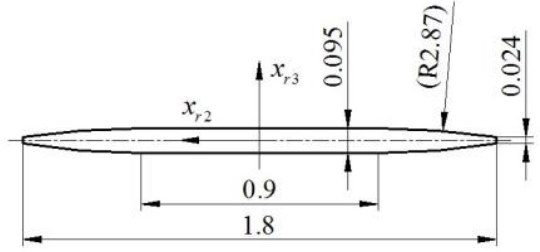

Figure 2

The cross section and material coordinate system of a flat roving

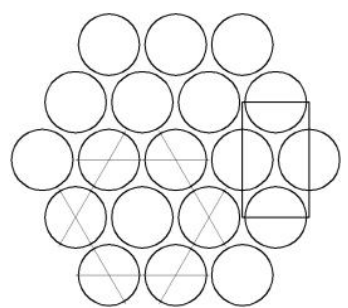

Figure 3

Hexagonal layout of the fibers in the roving

In the studied case the matrix is always isotropic, but the carbon fibers are transversely isotropic and are in a hexagonal layout in the roving model-cell, therefore, also the roving-model cell has transversely isotropic behavior. In the roving the plane of isotropy is the $x_{r 2} x_{r 3}$ plane. For the investigated case the macroscopic material properties determined by the roving model-cell are [3]:

$$
\begin{array}{lll}
E_{r 1}=177236 \mathrm{MPa}, & v_{r 12}=v_{r 13}=0.202, & G_{r 12}=G_{r 13}=4115 \mathrm{MPa}, \\
E_{r 2}=E_{r 3}=10352 \mathrm{MPa}, & v_{r 23}=0.430, & G_{r 23}=3620 \mathrm{MPa} .
\end{array}
$$

We validate the results of the roving model-cell also here because these material properties are the input data for the textile composite layer model-cell. The experiment validates both the results of the roving model-cell and the textile composite layer model-cell together. 


\section{The Layer Model-Cell}

\subsection{The Geometry and Finite Element Mesh of Layer Model- Cell}

A textile composite layer can also be considered as periodic parts, volume elements, cells. The layer can be built up from these periodic domains, from so called model-cells.

We show the building up of the layer model-cell, and validate the numerical results by experiment. We produced the eight layer, $2 \mathrm{~mm}$ thick composite specimen reinforced with plain weave carbon textile used for the experiments by manual lamination (Figure 4). The matrix material was polyester resin. In order to prevent that the production process would influence the material properties of the test specimens we hardened each sheet with identical technological parameters in the autoclave: $T=65-68^{\circ} \mathrm{C}, p=6 \mathrm{bar}, t=2 \mathrm{~h}$.

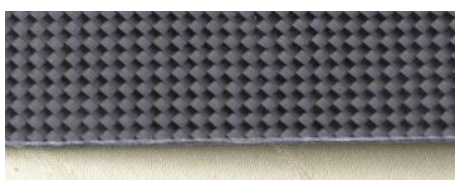

Figure 4

Eight-layer textile composite plate

The thickness of the textile and the waviness of the roving we determined after lamination by measurement. The cross section of the textile can be seen in Fig. 5 after lamination.

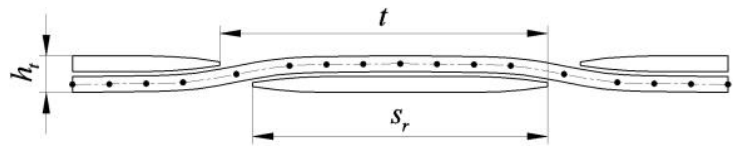

Figure 5

Cross section of the investigated textile after lamination

During the building up of the layer model with thickness $h_{t}$ we assumed that the textile with $h_{t}$ thickness is located in the middle of the composite layer (Fig. 6):

$v=\frac{h-h_{t}}{2}$. 


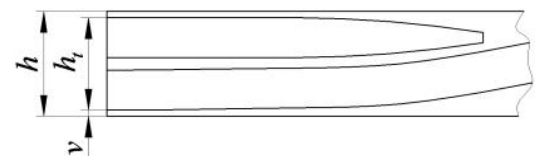

Figure 6

Textile in the composite layer

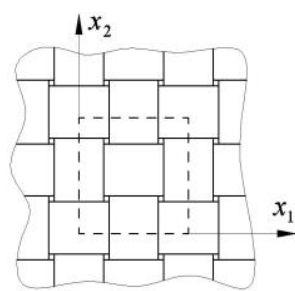

Figure 7

One unit in the textile

In order to be able to provide more simple boundary conditions we apply the side surfaces of the model cell parallel to the planes of the material principal directions [8]. The dimensions in $x_{1}$ and $x_{2}$ direction of the layer model-cell we determined in a way that the cell should contain one unit both in chain- and weft directions. In the case of a plain weave textile this means $2-2$ complete $(1+2 \times 0.5=2)$ roving (Figure 7, dashed line square). E.g. at a $2 / 2$ twill weave textile one unit consists of 4-4 roving.

The $x_{1}, x_{2}, x_{3}$ material principle directions of the model-cell and the $x_{r 1}, x_{r 2}, x_{r 3}$ principal direction of the roving can be seen in Figure 8. In the case of the roving $x_{r 1} x_{r 3}$ is the symmetry plane. We applied the model-cell's side surfaces perpendicular to $x_{1}$ and $x_{2}$ axes in a way that those should coincide with the $x_{r 1} x_{r 3}$ and $x_{r 2} x_{r 3}$ material principal direction planes of the roving. In this case the four side surfaces at tension remain in plane and they displace parallel with the $x_{1} x_{3}$ and $x_{2} x_{3}$ planes of the model-cell. At shear in $x_{1} x_{2}$ plane the points of the side surfaces will displace in the same way in direction of the shear. At shear, there is no deformation on the side surfaces perpendicular to $x_{1}$ axis in $x_{2}$ direction and on the surfaces perpendicular to $x_{2}$ axis in $x_{1}$ direction.

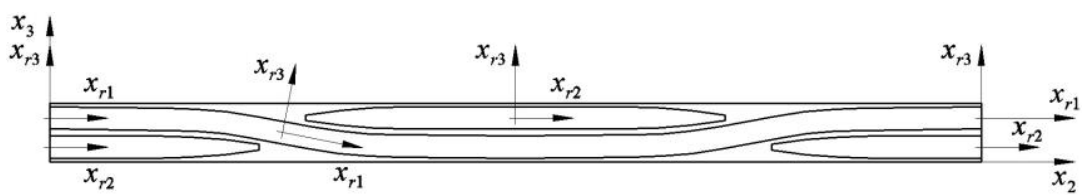

Figure 8

$x_{1}, x_{2}, x_{3}$ material principal direction of the layer model-cell and

$x_{r 1}, x_{r 2}, x_{r 3}$ material principal direction of the roving

The dimensions of the layer model-cell and the identity signs of the six side surfaces are seen in Figure 9. We marked the side lengths with $a$ and $b$ because we provided a general formulation for the composite layer finite element modeling. This formulation is also valid for such a weave where $a \neq b$, e.g. the chain- and 
the weft direction roving densities are not identical. The dimensions of the investigated composite layer model-cell in Figure 9 are:

$$
\begin{aligned}
& h=0.25 \mathrm{~mm}, h_{t}=0.22 \mathrm{~mm}, v=0.015 \mathrm{~mm}, \\
& a=b=4 \mathrm{~mm}, t_{1}=t_{2}=2 \mathrm{~mm}, s_{r 1}=s_{r 2}=1.8 \mathrm{~mm}, \\
& A_{A+}=A_{A-}=1 \mathrm{~mm}^{2}, A_{B+}=A_{B-}=1 \mathrm{~mm}^{2}, A_{C+}=A_{C-}=16 \mathrm{~mm}^{2} .
\end{aligned}
$$

We did the finite element computation with the NX I-deas 6.1 program code. We applied a mesh of parabolic tetrahedron elements for the layer model-cell. Due to the periodicity, we need to apply the so called periodical boundary conditions on the layer model-cell. Therefore, we need to generate the finite element mesh in a way that, on the opposite side surfaces there should be nodes opposite of each other and by this way node pairs will be created on two side surfaces of the model-cell. On the side surfaces perpendicular to $x_{1}$ and $x_{2}$ axes it was necessary to specify surface connections for the two-two surface pairs belonging to the matrix and located opposite to each other, and for the surface pair perpendicular to the $x_{3}$ axis.
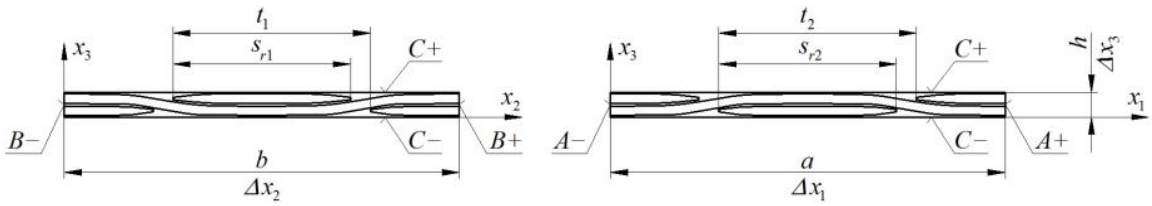

Figure 9

Dimensions of the layer model cell

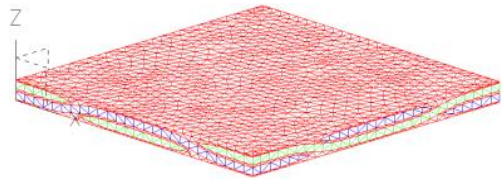

Figure 10

Finite element mesh of the layer model-cell

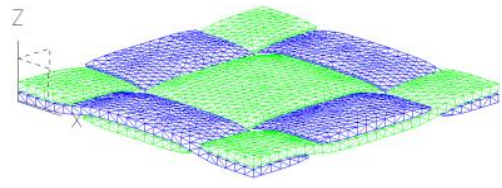

Figure 11

Finite element mesh of the textile layer

We generated the finite element mesh so that we generated second order triangle shaped shell elements for the surfaces first then by using those we generated second order tetrahedron shaped (3D) elements inside the layer model-cell. In Figure 10 we can see the finite element mesh of the layer model cell, which includes 38974 elements and 56587 nodes. Figure 11 shows the finite element mesh of the textile. $X Y Z$ coordinate system shown in Figures 10 and 11 corresponds to the $x_{1} x_{2} x_{3}$ coordinate system of Figures 8 and 9 . 


\subsection{Loading of the Layer Model-Cell}

We simulated the basic experiments of mechanics of materials with the layer model-cell in a way that we specified a kinematic loading and node displacement field for the side surfaces of the model-cell. For the nodes at the corners of the model-cell and at the center points of the side surfaces we referred with node numbers in Figure 12.
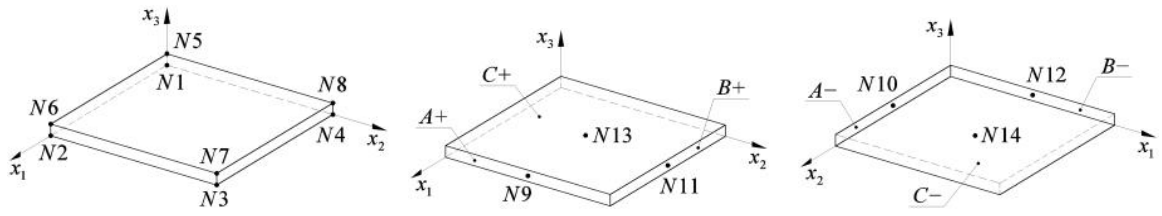

Figure 12

Marking the nodes at the eight corners of the layer model cell and at the center points of the side surfaces

A node displacement vector is in the model-cell in the $x_{1} x_{2} x_{3}$ coordinate system:

$$
\vec{u}=u \vec{e}_{1}+v \vec{e}_{2}+w \vec{e}_{3} .
$$

For the layer model-cell (Figure 9) the following general periodic boundary conditions can be applied [11]:

$u_{i}^{j+}\left(X_{1}, X_{2}, X_{3}\right)-u_{i}^{j-}\left(X_{1}, X_{2}, X_{3}\right)=\Delta x_{j} \bar{\varepsilon}_{i j}=c_{i}^{j}$.

$u_{i}^{j+}$ and $u_{i}^{j-}$ are the displacement in $X_{i}$ direction on the surface pair perpendicular to the $X_{j}$ axis in the $X_{1} X_{2} X_{3}$ global coordinate system. The $j+$ index means the positive $X_{j}$ axis and $j$ - marks the negative $X_{j}$ direction in the coordinate system of the model-cell (Figure 9). $\Delta x_{j}$ is the side length of the model-cell. $c_{i}^{j}$ ( $i=j=1,2,3$ ) is the change of distance of the side surfaces (tension or compression) in $X_{j}$ directions of the model-cell, whereas $c_{i}^{j}=c_{j}^{i}(i \neq j=1,2,3)$ is the displacement in the plane of the side surface resulting from the shear of the side surfaces. The (6) boundary condition ensures the periodicity and the continuity of the displacement field in the composite material layer. The (6) equation provides, for the appropriate points, the difference of the displacements on both opposite surfaces. $u_{i}^{j+}$ and $u_{i}^{j-}$ displacements are functions of $X_{1}, X_{2}, X_{3}$ coordinates. Therefore, these surfaces remain not necessarily in plane during the deformation.

With the layer model-cell we simulated the basic experiments (tension-pressure and shear) of mechanics of materials and we determined the orthotropic material properties from the model-cell computed stress and strain state. We realised the simulation of these basic experiments with separate, kinematic loading cases. 
During providing the kinematic loading, on $A+, A-, B+, B$ - side surfaces we gave displacements not for the individual nodes but the complete side surfaces in a way that we prescribed the displacement only for the middle node and we connected all nodes on the surface in the given direction. This prescription ensures that the side surface can displace in the prescribed direction as a rigid body. From the loading, we got reaction forces. These reaction forces were reduced to the center node of the surface. Since, the side surfaces of the layer model-cell coincide with the planes of the material principal directions of the roving (Figure 8), the following average stress appears on the side surfaces of the model-cell:

$\bar{\sigma}_{i j}=\frac{1}{A_{j}} \int_{\left(A_{j}\right)} \sigma_{i j} d A=\frac{F_{i}}{A_{j}}$.

$F_{i}$ is a reaction force in $x_{i}$ direction on the surface perpendicular to $x_{j}$ axis and, $A_{j}$ is the area of the side surface.

\subsection{Tension Test Simulation with the Layer Model-Cell}

For the computation of the $E_{1}$ modulus of elasticity and $v_{12}$ Poisson's ratio of the textile composite layer we need to simulate an $x_{1}$ directional tension for $E_{2}$ and for $v_{21}$ we need to simulate an $x_{2}$ directional tension with the layer model-cell. At tension the strains are non-zero $\left(\bar{\varepsilon}_{1}, \bar{\varepsilon}_{2} \neq 0\right)$ in the strain tensor in the (1) constitutive equation. The (6) general equation for tension is the following:

$$
c_{i}^{j}=\Delta x_{j} \bar{\varepsilon}_{j}, \quad \bar{\varepsilon}_{j}=\frac{c_{i}^{j}}{\Delta x_{j}}, \quad(i=j=1,2) .
$$

At tension tests the side surfaces of the model-cell perpendicular to $x_{1}$ and $x_{2}$ axes remain in plane and displace parallel with the planes of principal directions. We ensured the parallelism requirement in the case of the tension loading by connecting all the nodes on the side surface in the direction perpendicular to the surface. These prescriptions are summarized by the relationships (9) - (10). According to equation (9) we connected all nodes on $A+$ surface in $x_{1}$ direction. The independent node is $N 2$, meaning that the displacement of all nodes on $A+$ surface are identical with displacement of the $N 2$ node in $x_{1}$ direction.

$$
\begin{array}{ll}
u_{A-}=u\left(0 ; x_{2} ; x_{3}\right)=u_{N 1}, & u_{A+}=u\left(a ; x_{2} ; x_{3}\right)=u_{N 2}, \\
v_{B-}=v\left(x_{1} ; 0 ; x_{3}\right)=v_{N 5}, & v_{B+}=v\left(x_{1} ; b ; x_{3}\right)=v_{N 8} .
\end{array}
$$

In relationships $(9)-(10)$ :

$0 \leq x_{1} \leq a, \quad 0 \leq x_{2} \leq b, \quad 0 \leq x_{3} \leq h$. 
The $A$ - and $B$ - side surfaces were clamped only in the central node in the direction perpendicular to the surface at the $x_{1}$ and $x_{2}$ directional tensions:

$$
u_{A-}=u_{N 10}=0, \quad v_{B-}=v_{N 12}=0 .
$$

As a result of the tension the waviness (Figure 8) in the roving decreases in direction of the tension and increases perpendicularly to the tension. Therefore, $C+$ and $C$ - side surfaces do not remain in plane. In order to restrain the rigid body displacement and rotation of the $C$ - side surface we have to prescribe zero displacement for the $N 1$ node in $x_{3}$ direction:

$w_{N 1}=0$.

The kinematic loading belonging to the $x_{1}$ and $x_{2}$ directional tension test of the layer model-cell were determined as follows. At the $x_{i}$ directional tension we prescribed the $\bar{\varepsilon}_{i}$ specific strain for the model-cell and computed the $x_{i}$ directional displacement of the side surface perpendicular to the $x_{i}$ direction from the (8) equation. In this case, we did not prescribe the cross contraction.

In the case of the $x_{1}$ directional tension $\bar{\varepsilon}_{1}$ is given, consequently:

$$
u_{A+}=u_{N 9}=a \bar{\varepsilon}_{1} \text {. }
$$

In the case of the $x_{2}$ directional tension $\bar{\varepsilon}_{2}$ is prescribed, out of which the displacement of the $B+$ side surface is:

$v_{B+}=v_{N 11}=b \bar{\varepsilon}_{2}$.

In the case of $x_{1}$ and $x_{2}$ directional tensions we need to specify periodic boundary condition in addition so that the opposite nodes should displace on the same way on the side surface. For fulfilment of this requirement the node pairs must be connected on the opposite $A-/ A+$ and $B-/ B+$ side surfaces, except the edges of the model-cell:

$$
\begin{aligned}
& A-/ A+: \quad v\left(0 ; x_{2} ; x_{3}\right)=v\left(a ; x_{2} ; x_{3}\right), \quad w\left(0 ; x_{2} ; x_{3}\right)=w\left(a ; x_{2} ; x_{3}\right), \\
& B-/ B+: \quad u\left(x_{1} ; 0 ; x_{3}\right)=u\left(x_{1} ; b ; x_{3}\right), \quad w\left(x_{1} ; 0 ; x_{3}\right)=w\left(x_{1} ; b ; x_{3}\right) .
\end{aligned}
$$

In relationships (16) - (17) and (19) - (26):

$$
0<x_{1}<a, \quad 0<x_{2}<b, \quad 0<x_{3}<h .
$$

On the layer model cell we prescribed periodic boundary condition for the $C-/ C+$ side surface in the plane of the side surface.

$$
C-/ C+: \quad u\left(x_{1} ; x_{2} ; 0\right)=u\left(x_{1} ; x_{2} ; h\right), \quad v\left(x_{1} ; x_{2} ; 0\right)=v\left(x_{1} ; x_{2} ; h\right) \text {. }
$$


With this boundary condition we ensured that bending will not occur at the tension of the layer due to the decrease or increase of waviness of the roving. The straight line connecting the node pairs so remained perpendicular to the middle surface before deformation.

As we have already written earlier, we intend to use this layer model-cell for the determination of material properties of a given layer of a multilayer textile composite plate. Therefore, we cannot prescribe periodicity in $x_{3}$ direction for the $C-/ C+$ side surface while modeling a layer of such a composite plate where every layer is the same (textile, layer thickness, fiber direction). Namely there is a very small likelihood that the textile reinforcement is positioned in the same way in the layers following each other in the composite plate. For this case a $\left[0^{\circ} / 0^{\circ} / 0^{\circ} / 0^{\circ}\right]$ layer composition textile composite plate is shown as an example in Figure 13. The dashed lines mark the boundaries of the layers.

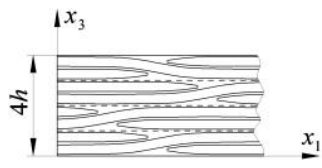

Figure 13

Positioning of the textile in a four layer composite plate

We need to connect the node pairs at the opposite edges, except the corner points of the model-cell, according to the (20) - (26) relationships:

On edges in $x_{1}$ direction: $u\left(x_{1} ; 0 ; 0\right)=u\left(x_{1} ; b ; 0\right), w\left(x_{1} ; 0 ; 0\right)=w\left(x_{1} ; b ; 0\right)$,

$$
\begin{aligned}
& u\left(x_{1} ; 0 ; h\right)=u\left(x_{1} ; b ; h\right), w\left(x_{1} ; 0 ; h\right)=w\left(x_{1} ; b ; h\right), \\
& u\left(x_{1} ; b ; 0\right)=u\left(x_{1} ; b ; h\right) .
\end{aligned}
$$

On edges in $x_{2}$ direction: $v\left(0 ; x_{2} ; 0\right)=v\left(a ; x_{2} ; 0\right), w\left(0 ; x_{2} ; 0\right)=w\left(a ; x_{2} ; 0\right)$,

$$
\begin{aligned}
& v\left(0 ; x_{2} ; h\right)=v\left(a ; x_{2} ; h\right), w\left(0 ; x_{2} ; h\right)=w\left(a ; x_{2} ; h\right), \\
& v\left(a ; x_{2} ; 0\right)=v\left(a ; x_{2} ; h\right) .
\end{aligned}
$$

On edges in $x_{3}$ direction: $w\left(0 ; 0 ; x_{3}\right)=w\left(a ; 0 ; x_{3}\right)=w\left(a ; b ; x_{3}\right)=w\left(0 ; b ; x_{3}\right)$.

On the $C$ - side surface every corner has the same displacement in $x_{3}$ direction. Therefore, we connect the nodes at the corners in $x_{3}$ direction. The independent node is $N 3$ :

$$
w_{N 1}=w_{N 2}=w_{N 4}=w_{N 3} \text {. }
$$


On the $C+$ side surface we also connect the nodes at the corners in $x_{3}$ direction. The independent node is $N 7$ :

$$
w_{N 5}=w_{N 6}=w_{N 8}=w_{N 7} .
$$

Figure 14 shows the connection between the nodes at the opposite side surfaces and edges. It is necessary to skip the edges at the opposite sides and the corners at the opposite edges so that the model-cell will not be over-determined.
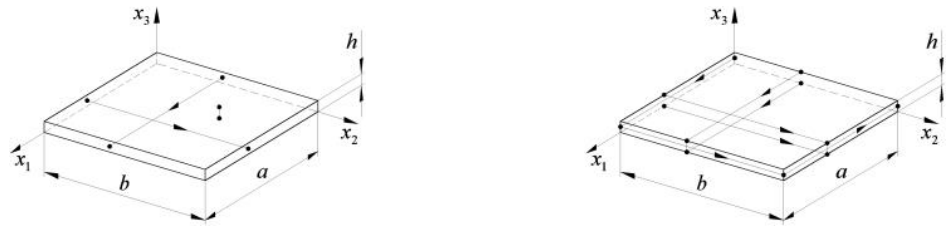

Figure 14

Connecting the nodes for ensuring the periodicity

At the tension in $x_{1}$ direction we numerically determined the following reaction forces and the cross contraction:

$$
\vec{F}_{A+}=-\vec{F}_{A-}, \vec{F}_{B-}=\vec{F}_{N 1}=\overrightarrow{0}, v_{B+} .
$$

We calculate the $\bar{\sigma}_{1}$ average normal stress as the quotient of the reaction force appearing on the $A+$ side surface and the area of the side surface according to (7). From that we determine $E_{1}$ Young's modulus in the usual way. $v_{12}$ Poisson's ratio can be calculated from the numerically determined $v_{B+}$ displacement and from the prescribed $u_{A+}$ displacement. These calculations can also be done for $x_{2}$ direction in the similar way. At the tension in $x_{2}$ direction we numerically determined the following reaction forces and the cross contraction:

$$
\vec{F}_{B+}=-\vec{F}_{B-}, \vec{F}_{A-}=\vec{F}_{N 1}=\overrightarrow{0}, u_{A+} .
$$

Table 1 summarises the prescribed kinematic loading of the investigated textile composite model cell as well as the numerically determined reaction forces, the cross contraction and the calculated average stresses and material properties for both loading cases.

Table 1

The given and determined quantities of the investigated textile composite model-cell

\begin{tabular}{|l|l|}
\hline \multicolumn{2}{|c|}{ Tension in $x_{1}$ direction } \\
\hline Kinematic loading & $\bar{\varepsilon}_{1}=2 \cdot 10^{-3}, u_{A+}=u_{N 9}=8 \cdot 10^{-3} \mathrm{~mm}$ \\
\hline \multirow{2}{*}{$\begin{array}{l}\text { Numerically determined } \\
\text { quantities }\end{array}$} & $\vec{F}_{A+}=\left(101.4 \vec{e}_{1}\right) \mathrm{N}, \vec{F}_{A-}=\left(-101.4 \vec{e}_{1}\right) \mathrm{N}$ \\
\cline { 2 - 2 } & $v_{B+}=-8.271 \cdot 10^{-4} \mathrm{~mm}$ \\
\hline
\end{tabular}




\begin{tabular}{|l|l|}
\hline Average stress & $\bar{\sigma}_{1}=101.4 \mathrm{MPa}$ \\
\hline Material properties & $E_{1}=50700 \mathrm{MPa}, v_{12}=0.103$ \\
\hline & Tension in $x_{2}$ direction \\
\hline Kinematic loading & $\bar{\varepsilon}_{2}=2 \cdot 10^{-3}, v_{B+}=v_{N 11}=8 \cdot 10^{-3} \mathrm{~mm}$ \\
\hline \multirow{2}{*}{$\begin{array}{l}\text { Numerically determined } \\
\text { quantities }\end{array}$} & $\vec{F}_{B+}=\left(101.4 \vec{e}_{2}\right) \mathrm{N}, \vec{F}_{B-}=\left(-101.4 \vec{e}_{2}\right) \mathrm{N}$ \\
\cline { 2 - 2 } & $u_{A+}=-8.271 \cdot 10^{-4} \mathrm{~mm}$ \\
\hline Average stress & $\bar{\sigma}_{2}=101.4 \mathrm{MPa}$ \\
\hline Material properties & $E_{2}=50700 \mathrm{MPa}, v_{21}=0.103$ \\
\hline
\end{tabular}

\subsection{Shear Test Simulation with the Layer Model-Cell}

Formula (6) of the pure shear looks as follows:

$$
c_{i}^{j}=\Delta x_{j} \frac{1}{2} \bar{\gamma}_{i j}, \quad c_{j}^{i}=\Delta x_{i} \frac{1}{2} \bar{\gamma}_{j i}, \quad(i \neq j=1,2) .
$$

For the calculation of $G_{12}$ shear modulus of elasticity of the textile composite layer we simulated a pure shear on the $x_{1} x_{2}$ surface. In this case the $\frac{1}{2} \bar{\gamma}_{12}=\frac{1}{2} \bar{\gamma}_{21}$ average shearing strain differs from zero in the strain tensor (1).

$\frac{1}{2} \bar{\gamma}_{12}=\frac{1}{2} \bar{\gamma}_{21}, \quad \bar{\gamma}_{12}=\frac{1}{2} \bar{\gamma}_{12}+\frac{1}{2} \bar{\gamma}_{21}$.

We modeled the pure shear by a prescribed displacement in $x_{2}$ direction of the side surfaces perpendicular to the $x_{1}$ axis or by a prescribed displacement in $x_{1}$ direction of the side surfaces perpendicular to the $x_{2}$ axis of the layer model-cell. We realized the above kinematic prescriptions/conditions at the center points of the $A+, A-, B+, B-$ side surfaces by using the following values:

$$
\begin{array}{ll}
v_{A-}=v_{N 10}=0, & v_{A+}=v_{N 9}=a \frac{1}{2} \bar{\gamma}_{21}, \\
u_{B-}=u_{N 12}=0, & u_{B+}=u_{N 11}=b \frac{1}{2} \bar{\gamma}_{12} .
\end{array}
$$

For the modeling of the shear test it was necessary to connect the nodes on the side surfaces perpendicular to $x_{1}$ and $x_{2}$ axes:

$$
v_{A-}=v\left(0 ; x_{2} ; x_{3}\right)=v_{N 1}, \quad v_{A+}=v\left(a ; x_{2} ; x_{3}\right)=v_{N 2},
$$




$$
u_{B-}=u\left(x_{1} ; 0 ; x_{3}\right)=u_{N 5}, \quad u_{B+}=u\left(x_{1} ; b ; x_{3}\right)=u_{N 8} .
$$

In relationships (33) $-(34)$ :

$0 \leq x_{1} \leq a, \quad 0 \leq x_{2} \leq b, \quad 0 \leq x_{3} \leq h$.

In the case of the $x_{1} x_{2}$ plane shear the $C+$ and $C$ - side surfaces do not remain in plane. For prevention of the rigid body displacement and rotation we restrained the $N 1$ node at the center of $C$ - side surface in $x_{3}$ direction:

$w_{N 1}=0$.

In the case of a pure shear the node pairs must be connected at the opposite side surfaces, with the exception of the edges of the model-cell, according to (37) (39). In the plane of $C-/ C+$ side surface, similarly to the tension test simulation, we also provided periodic boundary condition.

$$
\begin{array}{lll}
A-/ A+: & u\left(0 ; x_{2} ; x_{3}\right)=u\left(a ; x_{2} ; x_{3}\right), & w\left(0 ; x_{2} ; x_{3}\right)=w\left(a ; x_{2} ; x_{3}\right) . \\
B-/ B+: & v\left(x_{1} ; 0 ; x_{3}\right)=v\left(x_{1} ; b ; x_{3}\right), & w\left(x_{1} ; 0 ; x_{3}\right)=w\left(x_{1} ; b ; x_{3}\right) . \\
C-/ C+: & u\left(x_{1} ; x_{2} ; 0\right)=u\left(x_{1} ; x_{2} ; h\right), & v\left(x_{1} ; x_{2} ; 0\right)=v\left(x_{1} ; x_{2} ; h\right) .
\end{array}
$$

In (37) - (39) and (41) - (47) relationships:

$$
0<x_{1}<a, \quad 0<x_{2}<b, \quad 0<x_{3}<h .
$$

In the case of the opposite edges, except of the corner points of the model-cell, we need to connect the node pairs as follows:

On edges in $x_{1}$ direction: $v\left(x_{1} ; 0 ; 0\right)=v\left(x_{1} ; b ; 0\right), w\left(x_{1} ; 0 ; 0\right)=w\left(x_{1} ; b ; 0\right)$,

$$
\begin{aligned}
& v\left(x_{1} ; 0 ; h\right)=v\left(x_{1} ; b ; h\right), w\left(x_{1} ; 0 ; h\right)=w\left(x_{1} ; b ; h\right), \\
& v\left(x_{1} ; b ; 0\right)=v\left(x_{1} ; b ; h\right) .
\end{aligned}
$$

On edges in $x_{2}$ direction: $u\left(0 ; x_{2} ; 0\right)=u\left(a ; x_{2} ; 0\right), w\left(0 ; x_{2} ; 0\right)=w\left(a ; x_{2} ; 0\right)$,

$$
\begin{aligned}
& u\left(0 ; x_{2} ; h\right)=u\left(a ; x_{2} ; h\right), w\left(0 ; x_{2} ; h\right)=w\left(a ; x_{2} ; h\right), \\
& u\left(a ; x_{2} ; 0\right)=u\left(a ; x_{2} ; h\right) .
\end{aligned}
$$

On edges in $x_{3}$ direction: $w\left(0 ; 0 ; x_{3}\right)=w\left(a ; 0 ; x_{3}\right)=w\left(a ; b ; x_{3}\right)=w\left(0 ; b ; x_{3}\right)$.

For taking into consideration the periodicity we connect the nodes at the corners in $x_{3}$ direction on $C$ - side surface, the independent node is $N 3$ :

$w_{N 1}=w_{N 2}=w_{N 4}=w_{N 3}$. 
On the $C+$ side surface we also connect the nodes at the corner points in $x_{3}$ direction, the independent node is the $N 7$ :

$w_{N 5}=w_{N 6}=w_{N 8}=w_{N 7}$.

The numerically determined reaction forces at $x_{1} x_{2}$ plane shear are the following: $\vec{F}_{A+}=-\vec{F}_{A-}, \vec{F}_{B+}=-\vec{F}_{B-}, \vec{F}_{N 1}=\overrightarrow{0}$.

Due to the duality of $\tau$ stresses the average shear stress must be identical on the side surfaces perpendicular to the $x_{1}, x_{2}$ axes:

$\bar{\tau}_{12}=\frac{F_{1 B+}}{A_{B+}}, \quad \bar{\tau}_{21}=\frac{F_{2 A+}}{A_{A+}}, \quad \bar{\tau}_{12}=\bar{\tau}_{21}$.

The stress distribution is never homogenous in the layer model-cell. Figure 15 shows the $\tau_{12}$ stress distribution, the deformation is illustrated with a zoom of $200 \times$.

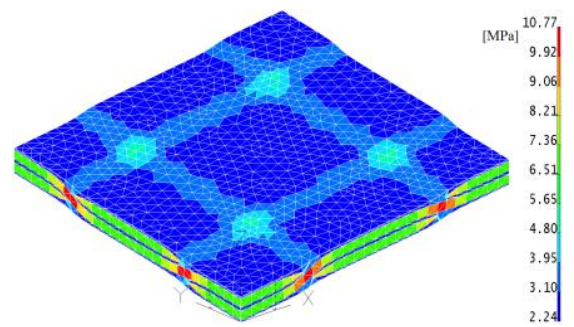

Figure 15

$\tau_{12}$ stress distribution in the layer model-cell at shear test in $x_{1} x_{2}$ plane

Shear modulus in $x_{1} x_{2}$ plane:

$G_{12}=\frac{\bar{\tau}_{12}}{\bar{\gamma}_{12}}$.

Table 2 summarises the given kinematic loading at the plane shear test of the textile composite model-cell as well as the numerically determined reaction forces, the average stresses and the shear modulus.

Table 2

The given and determined quantities of the investigated textile composite model-cell

\begin{tabular}{|l|l|}
\hline \multicolumn{2}{|c|}{ Pure shear in the $x_{1} x_{2}$ plane } \\
\hline \multirow{2}{*}{$\begin{array}{l}\text { Kinematic } \\
\text { loading }\end{array}$} & $\bar{\gamma}_{12}=2 \cdot 10^{-3}, \frac{1}{2} \bar{\gamma}_{12}=\frac{1}{2} \bar{\gamma}_{21}=10^{-3}$ \\
\cline { 2 - 2 } & $v_{A+}=v_{N 9}=4 \cdot 10^{-3} \mathrm{~mm}, u_{B+}=u_{N 11}=4 \cdot 10^{-3} \mathrm{~mm}$ \\
\hline
\end{tabular}




\begin{tabular}{|l|l|}
\hline \multirow{2}{*}{ Reaction forces } & $\vec{F}_{A+}=\left(5.8 \vec{e}_{2}\right) \mathrm{N}, \vec{F}_{A-}=\left(-5.8 \vec{e}_{2}\right) \mathrm{N}$ \\
\cline { 2 - 3 } & $\vec{F}_{B+}=\left(5.8 \vec{e}_{1}\right) \mathrm{N}, \vec{F}_{B-}=\left(-5.8 \vec{e}_{1}\right) \mathrm{N}$ \\
\hline Average stresses & $\bar{\tau}_{12}=5.8 \mathrm{MPa}, \bar{\tau}_{21}=5.8 \mathrm{MPa}$ \\
\hline Shear modulus & $G_{12}=2900 \mathrm{MPa}$ \\
\hline
\end{tabular}

\subsection{Macroscopic Material Properties of the Composite Layer Determined by the Layer Model-Cell - Summary of Numerical Results}

According to Sections 3.3 and 3.4, by carrying out simulations on the layer modelcell five orthotropic material constants can be defined, four of them are independent due to the requirements of (2) equation. However, in this case the studied composite layer has only three independent material constants because the reinforcement in the textile is the same in $x_{1}$ and $x_{2}$ directions (Figure 9):

$$
E_{1}=E_{2}=50700 \mathrm{MPa}, \quad v_{12}=0.103, \quad G_{12}=2900 \mathrm{MPa} .
$$

\subsection{Characteristic Data of the Textile Composite Layer Used in the Experiment}

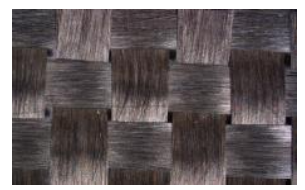

Figure 16

Plain weave textile

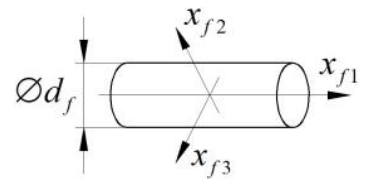

Figure 17

Material coordinate system of a carbon fiber

The composite is reinforced with a SIGRATEX KDL 8003 type plain weave carbon tissue. In Figure 16 we can see the plain weave textile before impregnation. The elementary fibers are ordered into a flat, untwisted roving. The type of the roving is Torayca T300-3K. $3 \mathrm{~K}$ means that there are $n_{f}=3000$ carbon fibers with $d_{f}=7 \mu \mathrm{m}$ in the roving.

Material properties of applied carbon fiber are known from the [7], [13] literature, in the $x_{f 1} x_{f 2} x_{f 3}$ material coordinate system of Figure 17:

$$
\begin{array}{lll}
E_{f 1}=230000 \mathrm{MPa} & v_{f 12}=v_{f 13}=0.166 & G_{f 12}=G_{f 13}=6432 \mathrm{MPa} \\
E_{f 2}=E_{f 3}=15000 \mathrm{MPa} & v_{f 23}=0.400 & G_{f 23}=5357 \mathrm{MPa} .
\end{array}
$$


The matrix material is an AROPOL M105TB type polyester resin, which is linear elastic; isotropic material. The $E_{m}$ Young's modulus and the $v_{m}$ Poisson's ratio of the matrix were determined by measurement according to ASTM D638-10 standard [1]. The $G_{m}$ modulus of elasticity was determined by the (53) relationship, that is valid for the isotropic materials:

$$
E_{m}=3677 \mathrm{MPa}, \quad v_{m}=0.346, \quad G_{m}=\frac{E_{m}}{2\left(1+v_{m}\right)}=1365.9 \mathrm{MPa} .
$$

We produced layered composite sheets also from the polyester resin, from which we cut out the test specimens which we needed for the measuring of the material properties of the matrix material. The parameters of production process of the matrix material were identical to the ones applied at the production of the textile composite plates $\left(T=65-68^{\circ} \mathrm{C}, p=6 \mathrm{bar}, t=2 \mathrm{~h}\right)$, therefore the material properties measured on these test specimen must be identical to the material properties of the matrix material of the investigated composite.

\section{Validation of Results of the Layer Model-Cell by Measurement}

The macroscopic material constants of a layer of an eight-layer textile composite laminate was determined by finite element simulation with the layer model-cell in Section 3. For validation of results by measurement we can only use an eight-layer composite laminate.

In the measurement investigated laminate all layers has the same orientation: $\left[0^{\circ} / 0^{\circ} / 0^{\circ} / 0^{\circ} / 0^{\circ} / 0^{\circ} / 0^{\circ} / 0^{\circ}\right]$. The $x_{1} x_{2} x_{3}$ material coordinate system of the composite laminate is identical to the material coordinate system of a single layer. The material constants of the eight-layer textile composite plate are identical to the material constants of the single layers (52), therefore:

$E_{1}^{8 l}=E_{2}^{8 l}=E_{1}=50700 \mathrm{MPa}, v_{12}^{8 l}=v_{12}=0.103, G_{12}^{8 l}=G_{12}=2900 \mathrm{MPa}$.

The $E_{1}$ modulus of elasticity and the $v_{12}$ Poisson's ratio of the eight-layer textile composite were determined by measurement according to EN ISO 527-4 standard [6]. The experimental examinations were performed with the test specimen that can be seen in Figure 18. In the experiment tensions took place in the material principal directions, $\vec{e}_{1}=\vec{e}_{x}$ and $\vec{e}_{2}=\vec{e}_{y}$. In the tension test the $\varepsilon_{1}$ longitudinal and the $\varepsilon_{2}$ transversal strain, the $F$ tension force and the change of $L$ length of specimen were measured with $t=0.02 \mathrm{sec}$ sampling. 


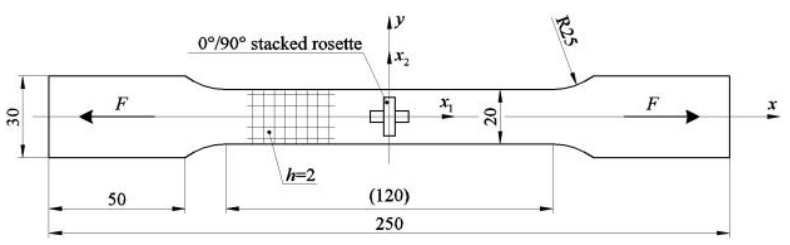

Figure 18

Tension test specimen

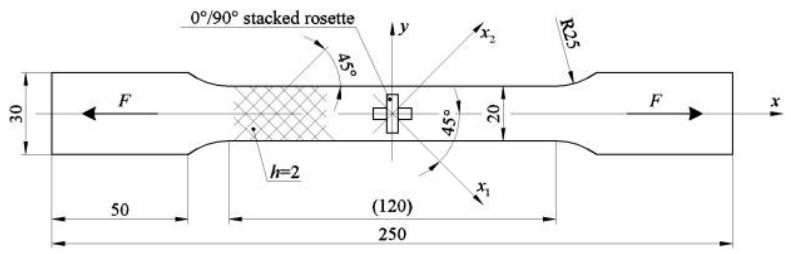

Figure 19

Shear test specimen

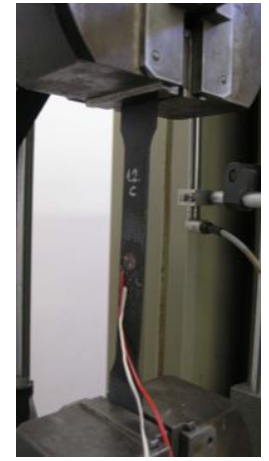

Figure 20

Test specimen in the tension test machine

For determination of $E_{1}$ modulus of elasticity a $\sigma_{1}\left(\varepsilon_{1}\right)$ linear function was fitted on the $\left(\varepsilon_{1} ; \sigma_{1}\right)$ measured point set by the least squares method. The slope of this linear function is the investigated $E_{1}$. For the determination of $v_{12}$ Poisson's ratio a $\varepsilon_{2}\left(\varepsilon_{1}\right)$ linear function was fitted for the $\left(\varepsilon_{1} ; \varepsilon_{2}\right)$ measured point set by the least squares method. $v_{12}$ is equal to the -1 times the slope of the obtained linear function. We processed the measurement data by the Microsoft Excel program. We carried out the tension test by using six tension test specimens. One of these can be seen in Figure 20 in the tension test machine.

The $G_{12}$ shear modulus was measured according to regulations of ASTM D3518 / D3518M - 13 standard [2]. The shape and dimension (Figure 19) of the shear test specimen was the same as the tension test specimen (Figure 18), however in the shear test specimen the reinforcing textile is positioned in every layer in $45^{\circ} \mathrm{com}$ pared to the longitudinal axis of the test specimen. It means that the $x_{1}$ material principal direction had a $-45^{\circ}$ angle with the axis of the tension. At the shear test the $\varepsilon_{x}, \varepsilon_{y}$ strains, the $F$ tension force and the change of $L$ length of specimen were measured with $t=0.02 \mathrm{sec}$ sampling.

The $\gamma_{12}$ shearing strain value from the $\varepsilon_{x}, \varepsilon_{y}$ strains (coordinate transformation) and the $\tau_{12}$ shear stress from the $\sigma_{x}$ normal stress could be computed. The $\tau_{12}$ stress is a linear function of the $\gamma_{12}$ shearing strain. For determination of $G_{12}$ shear modulus of elasticity a $\tau_{12}\left(\gamma_{12}\right)$ linear function was fitted on the $\left(\gamma_{12} ; \tau_{12}\right)$ measured point set by the least squares method. The slope of this linear function is 
the investigated $G_{12}$. The shear test was also carried out by using six test specimens.

The averages of the material constants were calculated from the six tension and six shear results. We determined the variance and variance square of the measured values as well as the deviation from the average. If the deviation was too large at any of the test specimens, we studied whether the given measurement has a mathematically or statistically detectable gross error. If we noted a gross error, we ignored the measured results belonging to the given experiment. Then we used Student's distribution to calculate the error range of the determined material constants [5].

Table 3 contains the computed and measured results for the $E_{1}$ modulus of elasticity, the $v_{12}$ Poisson's ratio and the $G_{12}$ shear modulus of elasticity. We can observe that the material properties created by the layer model-cell are within the margin of error of the measurement. The deviation of computed results is below $4 \%$ compared to the average of the measurement. So, the layer model-cell fulfils the necessary accuracy required for engineering modeling. This fact confirms that the model-cell approach for determination of layer properties is an efficient and well applicable method for determination of the orthotropic material constants of textile composite layers.

Table 3

Comparing the measurement results to the layer model-cell results

\begin{tabular}{|l|l|l|c|}
\hline $\begin{array}{c}\text { Finite element } \\
\text { layer model-cell }\end{array}$ & \multicolumn{1}{|c|}{ Measurement } & \multicolumn{2}{|c|}{$\begin{array}{c}\text { Deviation of the results of } \\
\text { model-cell from the average } \\
\text { value of measurement }\end{array}$} \\
\hline$E_{1}=50700 \mathrm{MPa}$ & $E_{1}=50094 \pm 1480 \mathrm{MPa}$ & $+606 \mathrm{MPa}$ & $+1.21 \%$ \\
\hline$v_{12}=0.103$ & $v_{12}=0.102 \pm 0.016$ & +0.001 & $+0.98 \%$ \\
\hline$G_{12}=2900 \mathrm{MPa}$ & $G_{12}=3018 \pm 254 \mathrm{MPa}$ & $-118 \mathrm{MPa}$ & $-3.91 \%$ \\
\hline
\end{tabular}

\section{Conclusions}

The paper presents a layer model-cell for the estimation and determination of the macroscopic, orthotropic material properties of a single layer of a multilayered textile composite material. It introduces the building up of the finite element layer model-cell and the prescription of boundary conditions applied to the finite element computations. It shows an example for determination of macroscopic material properties of the given textile composite layer with the numerical modeling of a unidirectional tension and plane shear by the layer model-cell.

Beside the determination of the material properties by the layer model cell the paper shows results for the same material by experimental way. The comparison of the results obtained from simulation and experiments confirms and proves the applicability of the layer model-cell and the roving model-cell in engineering. 


\section{References}

[1] ASTM D638-10, Standard Test Method for Tensile Properties of Plastics, 2010

[2] ASTM D3518 / D3518M - 13 Standard Test Method for In-Plane Shear Response of Polymer Matrix Composite Materials by Tensile Test of a $\pm 45^{\circ}$ Laminate, 2013

[3] Bojtár G., M. Csizmadia B., Égert J.: Numerical Estimation Method of Orthotropic Material Properties of a Roving for Reinforcement of Composite Materials, Acta Polytechnica Hungarica, Vol. 13, No. 6, p. 163182, ISSN 1785-8860, 2016

[4] Bunsell, A. R.; Renard, J.: Fundamentals of Fibre Reinforced Composite Materials, Series in Material Science and Engineering, Institute of Physics Publishing, 398 p., 2005

[5] M. Csizmadia B.: Planning Experiments, SZIE Egyetemi Kiadó, Gödöllő, 2016 (In Hungarian)

[6] EN ISO 527-4: Plastics - Determination of tensile properties - Part 4: Test conditions for isotropic and orthotropic fibre-reinforced plastic composites, 1997

[7] Goda T.: Wear Mechanism of Composite-Steel Sliding Pairs, PhD Thesis, 2002, Budapest University of Technology and Economics, Faculty of Mechanical Engineering, Supervisor: Váradi K. (In Hungarian)

[8] Hallal, A., Fardoun, F., Younes, R., Hage Chehade, F.: Evaluation of Longitudinal and Transversal Young's Moduli for Unidirectional Composite Material with Long Fibers, Advanced Materials Research, Vol. 324, p. 189192, 2011

[9] Kollár L. P.; Springer, G.: Mechanics of Composite Structures, Cambridge University Press, 480 p., 2003

[10] Kroupa, T., Janda, P., Zemčík, R.: Linear Two-Scale Model for Determining the Mechanical Properties of a Textile Composite Material, Materials and Technology, Vol. 46, No. 2, p. 97-101, 2012

[11] Leijan H.: Determining Micro- and Macro- Geometry of Fabric and Fabric Reinforced Composites, Dissertation, 2013, Department of Mechanical and Nuclear Engineering College of Engineering, Kansas State University, Manhattan, Kansas, Advisor: Youqi W.

[12] Siva Bhaskara Rao Devireddy; Sandhyarani Biswas: Effect of Fiber Geometry and Representative Volume Element on Elastic and Thermal Properties of Unidirectional Fiber-Reinforced Composites, Hindawi Publishing Corporation Journal of Composites, Article ID 629175, 12 pages, 2014 
[13] Torayca T300 Factory catalogue (No. CFA-001) Toray Carbon Fibers America, Inc. (CFA), 2012

[14] Xia, Z., Zhang, Y., Ellyin, F.: A Unified Periodical Boundary Conditions for Representative Volume Elements of Composites and Applications, International Journal of Solids and Structures, Vol. 40, No. 8, pp. 19071921,2003

[15] Zako, M., Uetsuji, Y., Kurashiki, T.: Finite Element Analysis of Damaged Woven Fabric Composite Materials, Composites Science and Technology, Vol. 63, Issues 3-4, p. 507-516, 2003

[16] Zhang C.: Multi-Scale Characterization and Failure Modeling of Carbon/Epoxy Triaxially Braided Composite, Dissertation, 2013, The Graduate Faculty of The University of Akron, Advisor: Binienda, W. K.

[17] Zhuo C.; Fan Y.; Meguid, S. A.: Multi-Level Modeling of Woven Glass/Epoxy Composite for Multilayer Printed Circuit Board Applications, International Journal of Solids and Structures, Vol. 51, Issues 21-22, pp. 3679-3688, 2014 